PROCEEDINGS OF THE

AMERICAN MATHEMATICAL SOCIETY

Volume 138, Number 11, November 2010, Pages 3957-3964

S 0002-9939(10)10374-8

Article electronically published on May 6, 2010

\title{
QUALITATIVE PROPERTIES OF ENTIRE RADIAL SOLUTIONS FOR A BIHARMONIC EQUATION WITH SUPERCRITICAL NONLINEARITY
}

\author{
ZONGMING GUO AND JUNCHENG WEI \\ (Communicated by Yingfei Yi)
}

\begin{abstract}
We study some qualitative properties of entire positive radial solutions of the supercritical semilinear biharmonic equation:

$$
\Delta^{2} u=u^{p} \text { in } \mathbb{R}^{n}, \quad n \geq 5, \quad p>\frac{n+4}{n-4} .
$$

It is known from a paper by Gazzola and Grunau that there is a critical value $p_{c}>(n+4) /(n-4)$ of $(*)$ for $n \geq 13$ and that $(*)$ has a singular solution $u_{s}(r)=K_{0}^{1 /(p-1)} r^{-4 /(p-1)}$. We show that for $5 \leq n \leq 12$ or $n \geq 13$ and $p<p_{c}$, any regular positive radial entire solution $u$ of $(*)$ intersects with $u_{s}(r)$ infinitely many times. On the other hand, if $n \geq 13$ and $p \geq p_{c}$, then $u(r)<u_{s}(r)$ for all $r>0$. Moreover, the solutions are strictly ordered with respect to the initial value $a=u(0)$.
\end{abstract}

\section{INTRODUCTION}

We consider some qualitative properties of entire positive radial solutions to the following supercritical biharmonic equation

$$
\Delta^{2} u=u^{p}, \quad u>0 \text { in } \mathbb{R}^{n}
$$

where $n \geq 5$ and $p>\frac{n+4}{n-4}$.

The corresponding supercritical second order elliptic equation

$$
-\Delta u=u^{p}, \quad u>0 \text { in } \mathbb{R}^{n}
$$

with $p>\frac{n+2}{n-2}$ and $n \geq 3$ was intensively studied. In particular, we mention the following theorem on the classification of positive radial entire solutions of (1.2).

Theorem 1.1 (Wang [4], Gui-Ni-Wang [3]). Let $n \geq 3$ and assume that $p>\frac{n+2}{n-2}$. Then for any $a>0$ the equation (1.2) admits a unique radial solution $u=u(r)$ such that $u(0)=a$ and $u(r) \rightarrow 0$ as $r \rightarrow+\infty$. The solution $u$ satisfies $u^{\prime}(r)<0$ for all $r>0$ and

$$
\lim _{r \rightarrow+\infty} r^{2 /(p-1)} u(r)=L:=\left[\frac{2}{p-1}\left(n-2-\frac{2}{p-1}\right)\right]^{1 /(p-1)} .
$$

Received by the editors August 5, 2009 and, in revised form, January 12, 2010

2010 Mathematics Subject Classification. Primary 35J30; Secondary 35B08, 35B33.

Key words and phrases. Entire radial solutions, biharmonic equations, singular solution. 
Moreover, if $n \leq 10$ or if $n \geq 11$ and

$$
p<p^{c}:=\frac{n^{2}-8 n+4+8 \sqrt{n-1}}{(n-2)(n-10)},
$$

then $u(r)-L r^{-2 /(p-1)}$ changes sign infinitely many times. If $n \geq 11$ and $p \geq p^{c}$, then $u(r)<L r^{-2 /(p-1)}$ for all $r>0$ and the solutions are strictly ordered with respect to the initial value $a=u(0)$.

The main purpose of this paper is to establish a similar theorem for entire solutions of (1.1).

Let us recall some known results on (1.1). In a recent paper 2, Gazzola and Grunau studied the existence and uniqueness of entire radial solutions to (1.1). They found the corresponding critical exponent $p_{c}$ for (1.1). To state their results, we first define $p_{c}$ to be the unique value of $p>\frac{n+4}{n-4}$ such that

$$
-(n-4)\left(n^{3}-4 n^{2}-128 n+256\right)(p-1)^{4}+128(3 n-8)(n-6)(p-1)^{3}
$$

$$
+256\left(n^{2}-18 n+52\right)(p-1)^{2}-2048(n-6)(p-1)+4096=0 .
$$

It has been shown in 2 that such a $p_{c}$ exists (and is unique) only when $n \geq 13$. Let

$$
u_{s}(r)=K_{0}^{1 /(p-1)} r^{-4 /(p-1)}
$$

where

$K_{0}=\frac{8}{(p-1)^{4}}\left[(n-2)(n-4)(p-1)^{3}+2\left(n^{2}-10 n+20\right)(p-1)^{2}-16(n-4)(p-1)+32\right]$.

It is easy to see that $u_{s}$ is a singular solution to (1.1) in $\mathbb{R}^{n} \backslash\{0\}$.

The main result in [2] is the following theorem.

Theorem $1.2([2])$. Let $n \geq 5$ and assume that $p>\frac{n+4}{n-4}$. Then for any $a>0$ the equation (1.1) admits a unique radial positive solution $u=u(r)$ such that $u(0)=a$ and $u(r) \rightarrow 0$ as $r \rightarrow+\infty$. Moreover, $u$ satisfies $u^{\prime}(r)<0, \Delta u(r)<0,(\Delta u)^{\prime}(r)>0$ for all $r>0$,

$$
\begin{gathered}
u(r)<\left(\frac{p+1}{2}\right)^{1 /(p-1)} u_{s}(r) \text { for all } r \geq 0, \\
\lim _{r \rightarrow+\infty} \frac{u(r)}{u_{s}(r)}=1 .
\end{gathered}
$$

Furthermore, for all $n \geq 13, p>p_{c}, u(r)-u_{s}(r)$ does not change sign infinitely many times.

In this paper we completely characterize the asymptotic behavior of the radial entire solutions of (1.1). We have the following theorem:

Theorem 1.3. Let $n \geq 5$ and assume that $p>\frac{n+4}{n-4}$. Then for any $a>0$ the equation (1.1) admits a unique radial solution $u=u(r)$ such that $u(0)=a$ and $u(r) \rightarrow 0$ as $r \rightarrow+\infty$. The solution u satisfies $u^{\prime}(r)<0$ for all $r>0$ and

$$
\lim _{r \rightarrow+\infty} r^{4 /(p-1)} u(r)=K_{0}^{1 /(p-1)} .
$$

Moreover, if $n \leq 12$ or if $n \geq 13$ and $p<p_{c}$, where $p_{c}$ is given by (1.5), then $u-K_{0}^{1 /(p-1)} r^{-4 /(p-1)}$ changes sign infinitely many times. If $n \geq 13$ and $p \geq p_{c}$, 
then $u(r)<K_{0}^{1 /(p-1)} r^{-4 /(p-1)}$ for all $r>0$ and the solutions are strictly ordered with respect to the initial value $a=u(0)$. Namely, if $u_{1}(r)$ and $u_{2}(r)$ are two radial solutions of (1.1) with $u_{1}(0)<u_{2}(0)$, then $u_{1}(r)<u_{2}(r)$ for $r>0$.

Since the existence and uniqueness of entire radial solution to (1.1) are already given by Theorem 1.2 we shall assume that $u_{a}$ is the unique entire radial solution of (1.1) with $u_{a}(0)=a$. If there is no confusion, we drop the index $a$.

In the rest of the paper, we proceed to prove Theorems 1.2 and 1.3. In Section 2, we collect some important preliminaries. In Section 3, we prove Theorem 1.2 , and in Section 4, we prove Theorem 1.3 .

After the completion of this paper, we came across the paper by Ferrero-GrunauKarageordis [1] in which they proved the first part of Theorem 1.3, i.e., when $p<p_{c}$. Their method, based on dynamical system, is quite different from ours. Our method generalizes the Sturm-Liouville comparison theorems to fourth order equations. In fact, our method in this paper in the case of $p \geq p_{c}$ also gives a new and more direct proof even in the second order case (Theorem 1.1).

\section{Emden-Fowler transformations, eigenvalues And SOME PRELIMINARIES}

As in 2], we use the Emden-Fowler transformation:

$$
u(r)=r^{-\frac{4}{p-1}} v(t), \quad t=\log r(r>0) .
$$

Therefore, after the change of (2.1), the equation in (1.1) may be rewritten as

$$
v^{(4)}(t)+K_{3} v^{\prime \prime \prime}(t)+K_{2} v^{\prime \prime}(t)+K_{1} v^{\prime}(t)+K_{0} v(t)=v^{p}(t), \quad t \in \mathbb{R}
$$

where the coefficients are given in [2, p. 911]. The characteristic polynomial (linearized at $\left.K_{0}^{1 /(p-1)}\right)$ is

$$
\nu \mapsto \nu^{4}+K_{3} \nu^{3}+K_{2} \nu^{2}+K_{1} \nu+(1-p) K_{0}
$$

and the eigenvalues are given by

$$
\begin{array}{ll}
\nu_{1}=\frac{N_{1}+\sqrt{N_{2}+4 \sqrt{N_{3}}}}{2(p-1)}, \quad \nu_{2}=\frac{N_{1}-\sqrt{N_{2}+4 \sqrt{N_{3}}}}{2(p-1)}, \\
\nu_{3}=\frac{N_{1}+\sqrt{N_{2}-4 \sqrt{N_{3}}}}{2(p-1)}, \quad \nu_{4}=\frac{N_{1}-\sqrt{N_{2}-4 \sqrt{N_{3}}}}{2(p-1)}
\end{array}
$$

where

$$
\begin{aligned}
N_{1}:= & -(n-4)(p-1)+8, \quad N_{2}:=\left(n^{2}-4 n+8\right)(p-1)^{2} \\
N_{3}:= & (9 n-34)(n-2)(p-1)^{4}+8(3 n-8)(n-6)(p-1)^{3} \\
& +\left(16 n^{2}-288 n+832\right)(p-1)^{2}-128(n-6)(p-1)+256 .
\end{aligned}
$$

Let us also define

$$
\tilde{\nu}_{j}=\nu_{j}-\frac{4}{p-1}, j=1,2,3,4 \text {. }
$$

A direct computation shows that $r^{\tilde{\nu}_{j}}$ are the four fundamental solutions to

$$
\Delta^{2} \psi=p u_{s}^{p-1} \psi
$$

Using Proposition 2 of [2] and direct verifications, we have the following proposition: 
Proposition 2.1. (i) For any $n \geq 5$ and $p>\frac{n+4}{n-4}$, we have

$$
\tilde{\nu}_{2}<2-n<0<\tilde{\nu}_{1} .
$$

(ii) For any $5 \leq n \leq 12$ or $n \geq 13, p<p_{c}$, we have $\tilde{\nu}_{3}, \tilde{\nu}_{4} \notin \mathbb{R}$ and $\operatorname{Re}\left(\tilde{\nu}_{3}\right)=$ $\operatorname{Re}\left(\tilde{\nu}_{4}\right)=\frac{4-n}{2}<0$.

(iii) For any $n \geq 13, p=p_{c}$, we have $\tilde{\nu}_{3}=\tilde{\nu}_{4}=\frac{4-n}{2}$.

(iv) For any $n \geq 13, p>p_{c}$, we have

$$
4-n<\tilde{\nu}_{4}<\frac{4-n}{2}<\tilde{\nu}_{3}<0, \quad \tilde{\nu}_{3}+\tilde{\nu}_{4}=4-n .
$$

Let us also recall the following theorem:

Theorem 2.2 ([2]). The following limits hold:

$$
\lim _{t \rightarrow+\infty} v(t)=K_{0}^{1 /(p-1)}, \quad \lim _{t \rightarrow+\infty} v^{(k)}(t)=0
$$

for any $k \geq 1$.

\section{THE CASE OF $p<p_{c}$}

In this section, we prove that for $p<p_{c}, u(r)-u_{s}(r)$ must have infinitely many intersections (and hence prove Theorem 1.2). This amounts to the study of the following linearized equation

$$
\Delta^{2} \phi=p u^{p-1} \phi, \quad \phi(r) \rightarrow 0 \text { as } r \rightarrow+\infty .
$$

First we have

Lemma 3.1. (1) If $\phi(0)=0$, then $\phi \equiv 0$.

(2) If $\phi(0)=1$, then $\Delta \phi(0)<0$.

Proof. (1) Suppose $\phi(0)=0$ and $\Delta \phi(0) \neq 0$. We may assume that $\Delta \phi(0)>0$. Since $\phi(0)=\phi^{\prime}(0)=0$, we may assume that $\phi(r)>0$ for $r \in(0, R)$ and $\phi(R)=0$. $\left(R\right.$ can be $+\infty$.) Then in $(0, R),(\Delta \phi)^{\prime}>0$, and hence $\Delta \phi(r)>0$ for $r \in(0, R)$. This implies that $\phi^{\prime}(r)>0$ and $\phi(r)>0$ for $r \in(0, R]$. This contradicts $\phi(R)=0$.

(2) follows from the same arguments.

As a consequence of (1) of Lemma 3.1, we have

Lemma 3.2. The solution to (3.1) is given by

$$
\phi(r)=c\left(\frac{4}{p-1} u(r)+r u^{\prime}(r)\right)
$$

for some $c \neq 0$.

The following theorem gives the asymptotic behavior of $u$, which is of independent interest.

Theorem 3.3. Let $u$ be the unique solution of (1.1). Then we have for $r$ large:

(3.3) $u(r)=\left(-K_{0}\right)^{-1 / 3} r^{-4 /(p-1)}+M_{1} r^{\alpha} \cos (\beta \ln r)+M_{2} r^{\alpha} \sin (\beta \ln r)+O\left(r^{\alpha-\delta}\right)$

where $\delta=\frac{\sqrt{N_{2}+4 \sqrt{N_{3}}}}{2(p-1)}>0, \nu_{3}=\alpha+\frac{4}{p-1}+i \beta$ and $M_{1}^{2}+M_{2}^{2} \neq 0$. (Note that it is known from Proposition 2 of [2] that $\alpha+\frac{4}{p-1}<0$.) 
Proof. Using the Emden-Fowler transformation,

$$
u(r)=r^{-\frac{4}{p-1}} v(t), \quad t=\log r(r>0),
$$

and letting $v(t)=\left(K_{0}\right)^{1 /(p-1)}-h(t)$, we see that $h(t)$ satisfies

$$
h^{(4)}(t)+K_{3} h^{\prime \prime \prime}(t)+K_{2} h^{\prime \prime}(t)+K_{1} h^{\prime}(t)+(1-p) K_{0} h(t)+O\left(h^{2}\right)=0, \quad t>1 .
$$

Therefore in the leading order, we can write

$$
h(t)=M_{1} e^{\left(\alpha+\frac{4}{p-1}\right) t} \cos \beta t+M_{2} e^{\left(\alpha+\frac{4}{p-1}\right) t} \sin \beta t+M_{3} e^{\nu_{2} t}+o\left(e^{\nu_{2} t}\right)
$$

(note that we have from Theorem 2.2 that $\lim _{t \rightarrow+\infty} h(t)=0$ ). This then implies that as $r \rightarrow+\infty$,

$$
\varphi(r)=M_{1} r^{\alpha} \cos (\beta \ln r)+M_{2} r^{\alpha} \sin (\beta \ln r)+M_{3} r^{\tilde{\nu}_{2}}+o\left(r^{\tilde{\nu}_{2}}\right)
$$

where $\varphi(r)=r^{-\frac{4}{p-1}} h(t):=u_{s}(r)-u(r)$.

We now show that $M_{1}^{2}+M_{2}^{2} \neq 0$.

Suppose that $M_{1}=M_{2}=0$. Then we have

$$
\varphi \sim r^{2-n-\kappa} \text { as } r \rightarrow+\infty
$$

where $\kappa=-\tilde{\nu}_{2}-(n-2)>0$ by Proposition 2.1. Furthermore, $\varphi(r)$ has no zeroes for $r$ large. We show that this is impossible. In fact, it is easy to see that $\varphi$ must change sign in $(0,+\infty)$. Otherwise, we assume $\varphi(r)>0$ for $r \geq 0$ (note that $u(r)<u_{s}(r)$ for $r$ small). Then using the behavior of $\varphi$ near $\infty$ and integrating the equation $\Delta^{2} \varphi=u_{s}^{p}(r)-u^{p}(r)$ over $\mathbb{R}^{n}$, we see that

$$
\int_{0}^{\infty}\left(u_{s}^{p}(r)-u^{p}(r)\right) r^{n-1} d r=0
$$

which contradicts $\varphi=u_{s}-u>0$. Here we need to use the fact that $p>(n+$ $4) /(n-4)$.

Suppose $\varphi(r)$ has exactly $k$ zeroes in $(0,+\infty)$ (recalling that $\varphi$ has no zeroes when $r$ is large) and $\varphi(r) \sim r^{2-n-\kappa}$ as $r \rightarrow+\infty$; we easily see that $r^{n-1} \varphi^{\prime}(r)$ has at least $k$ zeroes. On the other hand, since the function $\eta(r):=r^{n-1} \varphi^{\prime}(r)$ satisfies $\eta(0)=0$ (note $p>(n+2) /(n-2))$ and $\eta(r) \rightarrow 0$ as $r \rightarrow+\infty$, we see that $\eta^{\prime}(r)$ has at least $k+1$ zeroes. Thus $\Delta \varphi(r)=\frac{1}{r^{n-1}} \eta^{\prime}(r)$ has at least $k+1$ zeroes. A similar idea implies that $r^{n-1}(\Delta \varphi)^{\prime}(r)$ has at least $k$ zeroes and $\left(r^{n-1}(\Delta \varphi)^{\prime}(r)\right)^{\prime}$ has at least $k+1$ zeroes. Therefore, $\Delta^{2} \varphi=\frac{1}{r^{n-1}}\left(r^{n-1}(\Delta \varphi)^{\prime}(r)\right)^{\prime}$ has at least $k+1$ zeroes. This contradicts our assumption that $\varphi$ has $k$ zeroes, since $\Delta^{2} \varphi=p \xi^{p-1} \varphi$, where $\xi(r) \in\left(\min \left\{u(r), u_{s}(r)\right\}, \max \left\{u(r), u_{s}(r)\right\}\right)>0$ for all $r>0$. This proves our claim and completes the proof of Theorem 3.3

\section{The CASE OF $p \geq p_{c}$}

In this section, we consider the case $p \geq p_{c}$. We prove the following two theorems:

Theorem 4.1. For $p \geq p_{c}$, we have $u(r)<u_{s}(r)$ for $r>0$.

Theorem 4.2. For $p \geq p_{c}$, the solution to (3.1) remains of constant sign; that is,

$$
\frac{4}{p-1} u+r u^{\prime}(r)<0 .
$$

The proofs of both theorems depend on the use of the comparison principle for fourth order equations.

We prove Theorem 4.2 first. 
Proof of Theorem 4.2. Assume that Theorem4.1 holds; i.e., $u(r)<u_{s}(r)$. Let $\phi(r)$ be a solution of (3.1). By Lemma 3.1 we may assume that $\phi(0)=1, \Delta \phi(0)<0$. Let $\psi(r)=r^{\tilde{\nu}_{4}}$. Then it is easy to see that

$$
\Delta^{2} \psi=p u_{s}^{p-1} \psi
$$

By Proposition 2.1, we have $\tilde{\nu}_{4}>4-n$. This implies that $\int_{B_{r}(0)} r^{-4}|\phi| \psi<+\infty$. So we can multiply (3.1) by $\psi$ and (4.2) by $\phi$ and integrate over $B_{r}(0)$ to obtain (4.3)

$$
\begin{aligned}
0=\int_{B_{r}(0)} p\left(u_{s}^{p-1}-u^{p-1}\right) \phi \psi & +\int_{\partial B_{r}(0)}\left[(\Delta \phi)^{\prime} \psi-\Delta \phi \psi^{\prime}\right]+\int_{\partial B_{r}(0)}\left[\Delta \psi \phi^{\prime}-(\Delta \psi)^{\prime} \phi\right] \\
& =I_{1}(r)+I_{2}(r)+I_{3}(r)
\end{aligned}
$$

where $I_{i}(r)$ are defined in the last equality.

Let us assume that there exist $r_{1}, r_{2} \in(0,+\infty]$ such that

$$
\phi(r)>0, r \in\left(0, r_{1}\right), \phi\left(r_{1}\right)=0, \quad \Delta \phi(r)<0, r \in\left(0, r_{2}\right), \Delta \phi\left(r_{2}\right)=0 .
$$

We divide our proof into three cases:

Case 1. $r_{1}=r_{2}$.

In this case, we take $r=r_{1}=r_{2}$. Then we have $I_{1}(r)>0, I_{2}(r) \geq 0, I_{3}(r) \geq 0$. The identity (4.3) gives a contradiction.

Case 2. $r_{2}<r_{1}$.

In this case, we take $r=r_{2}$. Then it is easy to see that $I_{1}\left(r_{2}\right) \geq 0, I_{2}\left(r_{2}\right)=$ $\int_{\partial B_{r_{2}}(0)}\left[(\Delta \phi)^{\prime} \psi-\Delta \phi \psi^{\prime}\right] \geq 0$. It remains to estimate $I_{3}\left(r_{2}\right)$.

To this end, we first show that $\Delta \phi>0$ for $r \in\left(r_{2}, r_{1}\right)$. In fact, since $\Delta^{2} \phi=$ $p u^{p-1} \phi>0$ in $\left(0, r_{1}\right)$, we see that $\Delta \phi$ must be positive for $r>r_{2}$ and near $r_{2}$. Suppose that there exists $r_{3} \leq r_{1}$ such that $\Delta \phi\left(r_{3}\right)=0$. Then we have $\Delta \phi>0, \Delta(\Delta \phi)>0$ in $\left(r_{2}, r_{3}\right)$. This is impossible, since $\Delta \phi$ must attain its maximum in $\left(r_{2}, r_{3}\right)$ where $\Delta(\Delta \phi) \leq 0$.

Now we consider the function $\Phi(r)=r^{n-1}\left(\Delta \psi \phi^{\prime}-(\Delta \psi)^{\prime} \phi\right)$. Its derivative is given by

$$
\begin{aligned}
\Phi^{\prime}(r) & =\left(r^{n-1} \phi^{\prime}(r)\right)^{\prime} \Delta \psi(r)-\left(r^{n-1}(\Delta \psi)^{\prime}(r)\right)^{\prime} \phi(r) \\
& =r^{1-n}\left[\Delta \phi(r) \Delta \psi(r)-\phi(r) \Delta^{2} \psi(r)\right]<0 \text { for } r \in\left(r_{2}, r_{1}\right) .
\end{aligned}
$$

(Here we have used the fact that $\Delta \psi<0$.) So $\Phi\left(r_{2}\right)>\Phi\left(r_{1}\right)=r_{1}^{n-1} \Delta \psi\left(r_{1}\right) \phi^{\prime}\left(r_{1}\right) \geq$ 0 . As a consequence, we have proved that $I_{3}\left(r_{2}\right)=r_{2}^{1-n} \int_{\partial B_{r_{2}}(0)} \Phi\left(r_{2}\right) \geq 0$. So again, we have $I_{1}\left(r_{2}\right)>0, I_{2}\left(r_{2}\right) \geq 0, I_{3}\left(r_{2}\right) \geq 0$, and this gives a contradiction to the identity (4.3).

Case 3. $r_{1}<r_{2}$.

The proof is similar to Case 2. In this case, we take $r=r_{1}$. Then it is easy to see that $I_{1}\left(r_{1}\right) \geq 0, I_{3}\left(r_{1}\right)=\int_{\partial B_{r_{1}}(0)}\left[\Delta \psi \phi^{\prime}\right] \geq 0$. It remains to estimate $I_{2}\left(r_{1}\right)$.

As before, we first show that $\phi(r)<0$ for $r \in\left(r_{1}, r_{2}\right)$. In fact, since $\Delta \phi<0$ in $\left(0, r_{2}\right)$, we see that $\phi$ must be negative for $r>r_{1}$ and near $r_{1}$. Suppose that there exists $r_{3} \leq r_{2}$ such that $\phi\left(r_{3}\right)=0$. Then we have $\Delta \phi<0, \phi<0$ in $\left(r_{1}, r_{3}\right)$. This is impossible, since $\phi$ must attain its minimum in $\left(r_{3}, r_{2}\right)$ where $\Delta \phi \geq 0$. 
Now we consider the function $\Psi(r)=r^{n-1}\left((\Delta \phi)^{\prime} \psi-\Delta \phi \psi^{\prime}\right)$. Its derivative is given by

$$
\begin{aligned}
\Psi^{\prime}(r) & =\left(r^{n-1}(\Delta \phi)^{\prime}(r)\right)^{\prime} \psi(r)-\left(r^{n-1} \psi^{\prime}(r)\right)^{\prime} \Delta \phi(r) \\
& =r^{1-n}\left[\Delta^{2} \phi(r) \psi(r)-\Delta \phi(r) \Delta \psi(r)\right]<0 \text { for } r \in\left(r_{1}, r_{2}\right) .
\end{aligned}
$$

So $\Psi\left(r_{1}\right)>\Psi\left(r_{2}\right)=r_{2}^{n-1}(\Delta \phi)^{\prime}\left(r_{2}\right) \psi\left(r_{2}\right) \geq 0$. As a consequence, we have proved that $I_{2}\left(r_{1}\right)=r_{1}^{1-n} \int_{\partial B_{r_{1}}(0)} \Psi\left(r_{1}\right) \geq 0$. So again, we have $I_{1}\left(r_{1}\right)>0, I_{2}\left(r_{1}\right) \geq 0$, $I_{3}\left(r_{1}\right) \geq 0$ and a contradiction to the identity (4.3). These contradictions imply that $\phi$ remains of constant sign, and this completes the proof.

Proof of Theorem 4.1. The proof is similar to that of Theorem 4.2. Let $\phi_{0}=$ $u_{s}(r)-u(r)$. Then it is easy to see that $\phi_{0}$ satisfies

$$
\Delta^{2} \phi_{0}=u_{s}^{p}-\left(u_{s}-\phi_{0}\right)^{p} \leq p u_{s}^{p-1} \phi_{0}, r>0 .
$$

Now let $\psi_{0}(r)=r^{\tilde{\nu}_{3}}$. Then by Proposition 2.1, $\tilde{\nu}_{3} \geq \frac{4-n}{2}$. Thus $\int_{B_{R}(0)} r^{-4}\left|\phi_{0}\right| \psi_{0} \leq$ $\int_{B_{R}(0)} r^{-4} r^{-4 /(p-1)} r^{(4-n) / 2}<+\infty$ since $p>\frac{n+4}{n-4}$. Thus the integral $u_{s}^{p-1} \phi_{0} \psi_{0}$ is integrable. Similar to (4.3), we have the following identity:

$$
\int_{\partial B_{r}(0)}\left[\left(\Delta \phi_{0}\right)^{\prime} \psi_{0}-\Delta \phi_{0} \psi_{0}^{\prime}\right]+\int_{\partial B_{r}(0)}\left[\Delta \psi_{0} \phi_{0}^{\prime}-\left(\Delta \psi_{0}\right)^{\prime} \phi_{0}\right] \leq 0 .
$$

Now note that $\phi_{0}>0, \Delta \phi_{0}<0$ for $r$ small. So we may assume (4.4). The case $r_{1}=r_{2}$ is easy to exclude. We just need to consider the case $r_{2}<r_{1}$. To this end, we first show that $\Delta \phi_{0}>0$ for $r \in\left(r_{2}, r_{1}\right)$. In fact, since $\Delta^{2} \phi_{0}=u_{s}^{p}-\left(u_{s}-\phi_{0}\right)^{p}>0$ in $\left(0, r_{1}\right)$, we see that $\Delta \phi_{0}$ must be positive for $r>r_{2}$ and near $r_{2}$. Suppose that there exists $r_{3} \leq r_{1}$ such that $\Delta \phi_{0}\left(r_{3}\right)=0$. Then we have $\Delta \phi_{0}>0, \Delta\left(\Delta \phi_{0}\right)>0$ in $\left(r_{2}, r_{3}\right)$. This is impossible, since $\Delta \phi_{0}$ must attain its maximum in $\left(r_{2}, r_{3}\right)$ where $\Delta\left(\Delta \phi_{0}\right) \leq 0$. The rest of the proof is exactly the same as before. We omit the details.

Theorem 4.2 yields very important estimates on the asymptotic behavior of $u$.

Corollary 4.3. (1) Assume that $p \geq p_{c}$. Then the set of of solutions $\left\{u_{a}(r)\right\}$ to (1.1) is well ordered. That is, if $a>b$, then $u_{a}(r)>u_{b}(r)$ for all $r>0$.

(2) If $p>p_{c}$, then we have the following asymptotic expansion for $u$ :

$$
u(r)=K_{0}^{1 /(p-1)} r^{-4 /(p-1)}+M_{1} r^{\tilde{\nu}_{3}}+O\left(r^{\max \left(2 \tilde{\nu}_{3}, \tilde{\nu}_{4}\right)}\right)
$$

where $M_{1} \neq 0$. If $p=p_{c}$, then we have the following asymptotic expansion for $u$ :

$$
u(r)=K_{0}^{1 /(p-1)} r^{-4 /(p-1)}+\left(M_{1}+M_{2} \log r\right) r^{\frac{4-n}{2}}+O\left(r^{4-n}\right) .
$$

Proof of Corollary 4.3, For (1), we note that $\phi=\frac{\partial u_{a}}{\partial a}$ satisfies (3.1) with $\phi(0)=$ $1>0$. By Theorem 4.2, $\phi>0$. Thus $u_{a}(r)>u_{b}(r)$ for $a>b$.

For $(2)$, if $p>p_{c}$, we have

$$
u(r)=K_{0}^{1 /(p-1)} r^{-4 /(p-1)}+M_{1} r^{\tilde{\nu}_{3}}+M_{2} r^{\tilde{\nu}_{4}}+O\left(r^{\max \left(2 \tilde{\nu}_{3}, \tilde{\nu}_{4}\right)}\right) .
$$

If $M_{1}=0$, then

$$
u(r)=K_{0}^{1 /(p-1)} r^{-4 /(p-1)}+O\left(r^{\tilde{\nu}_{4}}\right),
$$

which implies that $\phi=O\left(r^{\tilde{\nu}_{4}}\right)$. Now as in the proof of Theorem 4.2, we have

$$
\int_{0}^{\infty} p\left(u_{s}^{p-1}-u^{p-1}\right) \phi r^{\tilde{\nu}_{4}} r^{n-1} d r=0
$$


where the integral is finite because $2 \tilde{\nu}_{4}<4-n$. This is impossible since $\phi>0$. So $M_{1} \neq 0$.

When $p=p_{c}$, (4.8) follows from the fact that $\tilde{\nu}_{3}=\tilde{\nu}_{4}=\frac{4-n}{2}$.

\section{ACKNOWLEDGMENTS}

The research of the first author is supported by a grant from NSFC (10571022). The research of the second author is partially supported by an Earmarked Grant from RGC of Hong Kong. The second author thanks Professor Nassif Ghoussoub for many useful discussions.

\section{REFERENCES}

[1] A. Ferrero, H.-Ch. Grunau and P. Karageorgis, Supercritical biharmonic equations with powerlike nonlinearity, Ann. Mat. Pura Appl. 188 (2009), 171-185. MR.2447933

[2] F. Gazzola and H.-Ch. Grunau, Radial entire solutions for supercritical biharmonic equations, Math. Ann. 334 (2006), 905-936. MR2209261 (2007b:35114)

[3] C. Gui, W. M. Ni and X. F. Wang, On the stability and instability of positive steady states of a semilinear heat equation in $\mathbf{R}^{n}$, Comm. Pure Appl. Math., Vol. XLV (1992), 1153-1181. MR.1177480 (93h:35095)

[4] X. F. Wang, On the Cauchy problem for reaction-diffusion equations, Trans. Amer. Math. Soc. 337 (1993), 549-590. MR1153016 (93h:35106)

Department of Mathematics, Henan Normal University, Xinxiang, 453007, People's Republic of China

E-mail address: guozm@public.xxptt.ha.cn

Department of Mathematics, The Chinese University of Hong Kong, Shatin, Hong KONG

E-mail address: wei@math.cuhk.edu.hk 